\title{
Behavior Analysis for Elderly
}

\author{
Albert Ali Salah ${ }^{1(凶)}$, Ben J.A. Kröse ${ }^{2,3}$, and Diane J. Cook ${ }^{4}$ \\ 1 Department of Computer Engineering, Boğaziçi University, Istanbul, Turkey \\ salah@boun.edu.tr \\ ${ }^{2}$ HvA University of Applied Sciences, Amsterdam, The Netherlands \\ b.j.a.krose@hva.nl \\ 3 University of Amsterdam, Amsterdam, The Netherlands \\ 4 School of Electrical Engineering and Computer Science, \\ Washington State University, Pullman, WA 99164-2752, USA \\ cook@eecs.wsu.edu
}

\begin{abstract}
Ubiquitous computing, new sensor technologies, and increasingly available and accessible algorithms for pattern recognition and machine learning enable automatic analysis and modeling of human behavior in many novel ways. In this introductory paper of the 6th International Workshop on Human Behavior Understanding (HBU'15), we seek to critically assess how HBU technology can be used for elderly. We describe and exemplify some of the challenges that come with the involvement of aging subjects, but we also point out to the great potential for expanding the use of ICT to create many applications to provide a better life for elderly.
\end{abstract}

\section{Introduction}

The aging population of the world calls for new applications, systems, and technologies, to be used by older adults, or for them. By elderly, we typically mean people of age 65 and above, although, of course, "old" is a fuzzy concept. In this overview paper, we focus on technology for people whose physical and cognitive skills are declining or impaired due to ageing, and particularly look at human behavior understanding for elderly.

Information and communication technology (ICT) serves a number of critical roles for elderly. The present volume primarily deals with analysis of older people's behaviors via systems and applications that ultimately target new services for elderly or their caretakers. But ICT is also conceived as a way of connecting the elder people to social life, as more and more people spend time in virtual worlds, and use them as a surrogate social life [43]. This function, however, requires not so much an invention of new sets of applications, but a re-thinking of the interfaces of existing applications so that elderly can be included. Indeed, it is reported by Selwyn and colleagues that age is a highly significant factor when it comes to the individual's ability to access and make use of ICT $[35,36]$. Subsequently, several issues are important in this context:

- How should technology interfaces be designed so that the elder people can have as much access to technology as possible? 
- What special technologies or applications should be implemented to improve the life of elderly?

- What guidelines, safeguards, and considerations should the development of new technology for elderly follow?

This overview is not an exhaustive attempt to answer all these questions, but rather an introduction for setting the stage and for describing recent research in this area. In Sect. 2, we describe the interaction and interface aspects, and also discuss some common applications for elderly. Section 3 deals with approaches of machine learning (and pattern recognition) for creating systems that adapt to elderly. Section 4 is on mobile solutions, where applications are ported to (or created for) smart phones. We conclude in Sect. 5 with a few observations about this rapidly developing field.

\section{Interactions with Elderly}

ICT effort for elderly focused in the last decade on the Ambient Assisted Living (AAL) vision, where smart environments provide services to elderly, especially in health monitoring and assistance [8]. AAL seeks to enable healthy and independent ageing of elderly, preferably in their home environment, where caregiving costs are reduced. In Europe, this is an important area of research, and the Ambient Assisted Living Association (AALA) is organizing the Ambient Assisted Living Joint Programme (AAL JP) ${ }^{1}$ to strenghten the ICT base for AAL. The recently completed "Support Action Aimed at Promoting Standards and Interoperability in the Field of AAL," within the auspices of AAL JP, promoted several representative use cases, which illustrate the focus of AAL research ${ }^{2}$ :

- Behavior Monitoring

- Calendar Service

- Social Interaction with Smart TV

- Shopping and Nutrition Planner

- Mobility Assistant

- Personal Trainer

- Environmental Health Monitoring and Alarms at Work

As will be obvious from this list, behavior understanding is a crucial part of the AAL vision, and finds applications from monitoring to personal trainers.

Jaschinski and Ben Allouch list the three general domains of AAL as ageing well at home, in the community, and at work, respectively [16]. Their work is conducted under the SONOPA (SOcial Networks for Older adults to Promote an Active life) project, which is a part of the 5th AAL JP Call termed 'ICTbased Solutions for (Self-)Management of Daily Life Activities of Older Adults at Home'. This project combines smart home technology and a social network

\footnotetext{
${ }^{1}$ http://www.aal-europe.eu/.

2 The final report available from http://www.aal-europe.eu/wp-content/uploads/ 2015/02/AAL_JP_Interop_D5_Final_Report.pdf.
} 
environment to create an integrated system which empowers applications like activity recognition or social matchmaking (for exchanging knowledge and services). SONOPA is one of the many AAL projects conducted in the last decade, and goes beyond the implementation of the individual technologies, which is a major theme in the earlier projects of the domain.

Among the many challenges of AAL, Jaschinski and Ben Allouch list the intrusiveness of the systems, protection and control issues over the collected data, the constant involvement of caregivers and family members (as a social burden), and the difficulty of replacing solutions in use with novel, more technologydependent solutions [16]. As can be seen from their analysis, these challenges are more social and cultural hurdles for the realization of AAL systems, as opposed to technological barriers (such as imperfect activity recognition systems), and require a strong design component.

This emphasis in interaction design is also apparent in the number of user studies devoted to measuring the quality of interaction of proposed smart systems for elderly. Sensor-based monitoring of the elderly, for instance the continuous detection of Activities of Daily Living (ADL) over long periods implies that a great amount of data should be collected, stored, processed and served to various applications. The creation of the ground truth for the training of learning systems is a costly process.

In another work performed under the SONOPA project, Eldib et al. propose the use of a network of visual sensors installed in an AAL environment to monitor the activities of an elderly over a long time [10]. The system is used to detect sleep patterns and bathroom visits, and subsequently can be used to find out about sleeping disorders. They use low-resolution visual sensors as opposed to a high-resolution camera (e.g. [14]), as a consideration for privacy concerns. They observe a single subject over ten months, and against a ground truth where the subject noted his sleep and wake up times in a diary, obtain good results for detecting the sleep patterns.

Such ground truth collection may not be possible for each observed subject and for every application scenario. While jotting down sleeping and waking times is not too much of an effort, other application scenarios may look at a broader set of activities, and thus require much more detailed ground truth annotation. Kanis et al. draw attention to the fact that the elderly needs to be empowered in terms of technology use, when it comes to applications and systems that fulfill the needs and respect the abilities of elderly [18]. They should know what kind of data are being collected, how they are used, and in general, "actively participate and contribute to sensing outcomes". Their approach is to record the data from a Living Lab, which is typically expensive to set up and operate, but allows much more realistic data collection over longer periods of time. These labs are equipped with various sensors, and allow a control center to select (and inspect) the data streams that are collected and stored at any given period. The people inhabiting the Living Lab for the duration of the experiment lead their normal lives (or a close approximation of it), and their daily activities are recorded. 
Ranjan and Whitehouse divide activities of daily living (ADL) into two broad categories, those that are performed in a home environment, and those that are performed in a community, respectively [29]. They continue listing a number of indices that can be used to measure an elderly persons ability to live and function within a home or a community. These measures are used in clinical settings, and while their terminology and extent sometimes differ, they all measure the frequency and the ability level in the assessed activities. Ranjan and Whitehouse remark that "Every state in US has its own set of ADLs, which is then applied uniformly to all elderly within the state," pointing out to the need for creating a dictionary of ADL labels that can be used widely. Because the performance of the elderly over ADLs has legal and clinical implications (including decisions to commit a person to a care facility, and insurance related consequences) the measurement of these sometimes require controlled conditions and involvement of certified experts. The behavior sensing technology, if to be used in such situations, should create reliable measurements for the experts' use.

On the other hand, depending on the purpose, monitoring the behavior of the elderly is sometimes performed in a very simple and straightforward way. In Japan, for instance, a special tea-kettle is sold, which can connect to the Internet and send a message everytime it is used, thus signalling the well-being or routineadherence of the elderly who uses it ${ }^{3}$. The specific cultural conditions lead to different technologies and solutions, making the design and the user interaction aspects essential. The assumptions under which behavior-sensing technology is presented to the elder should be different from those created for the contemporaries of the technology, and as such, should be validated via extensive user testing.

The cultural values shape the design, no matter how advanced the technology. An example that readily comes to mind is the SnowGlobe, designed by Visser et al., which can be placed in the house of the elder and its twin in the house of the loved ones [42]. The SnowGlobe displays movement of a remote user by glowing brighter, and users can exchange nudges by shaking their SnowGlobes, making the remote SnowGlobe blink. Subsequently, the device can increase the social connectedness of the elder by indicating presence and by displaying a visible call for attention only very briefly, so as not to disturb the daily life of the loved ones. This kind of concern is factored in by observing the cultural values and needs. Other forms of adaptation are achieved by designing systems that can learn over time.

\section{Learning Behavior Patterns}

Learning activities and behaviors is essential for pervasive systems, yet it is a challenging problem, because of the broadness of the domain, the possibilities of interleaving behaviors, and the inherent ambiguity in labeling the boundaries of behaviors [19].

\footnotetext{
${ }^{3}$ Internet-Connected Tea Kettle, http://www.mimamori.net/index.html.
} 
Simple behavior monitoring systems may be built on rules and fixed thresholds. In [24], a smarthome system is proposed to assist caregivers of dementia patients. Video cameras, motion sensors (and their combinations to detect postures and fall), contact sensors (for the doors and the beds), and inexpensive smart carpets are used to detect presence and track the people in the environment. The detection of anomalies (including falls and too long time spent in bathroom) follows a set of manually designed rules with adjustable thresholds. The availability of cheap sensors enables off-the-shelf solutions for simple activity detection, with little need for incorporating machine learning for adaptation.

Learning is useful when the analyzed activities are rich enough to exhibit idiosyncratic variations, and when the detection of an activity is not trivial with the available sensor configuration. In such cases, learning is used to improve detection and prediction rates.

In the work of Derungs et al., stroke patients' activity patterns and habits during their residence in a day-care center was learned based on motion data recordings from wearable inertial measurement units [9]. For such patients, it is very useful to quantify the amount of motion primitives like sitting, standing and walking, over a defined period of therapy, as the therapist will be able to see periods of positive and negative trends. In their study, Derungs et al. suggest extending the motion sensors with contextual information encoded by light, temperature, acoustic and vital sign sensors. This would not only help automatic classification, but also allow greater insight into activities performed by the patient in different contexts.

The effect of context can be incorporated into different approaches in different ways. Selecting sensors that can use such information and feeding additional data streams to the classifiers is but one way. In the contribution of Lago et al., the notion of a contextualized sequential pattern is introduced. This approach extends prefix trees for event-based sequential behavior mining [20].

In sequential pattern mining, frequently co-occuring patterns are detected from a sequence of events [23]. In an AAL context, these may be behaviors of an elder, recorded and classified into discrete events, like eating, working, washing, and such. Traditional sequence mining approaches like the Apriori algorithm [1] look at groups of events (or items) that occur together. Markov model based approaches, on the other hand, try to predict the next activity in the sequence, and require that the temporal dimension should also be discretized [19]. To alleviate the problem of detecting interleaved or concurrent events, conditional random fields can be used [39], but the temporal discretization is still a problem. The T-pattern approach, and its extensions, model the arrival times of events based on other, related events, and construct behavior dictionaries [22,33], thus allowing for flexible event intervals.

These approaches enable the discovery of behavior patterns that occur frequently, but also the detection of deviations from expected behaviors. Taking the temporal dimension into account makes it possible to investigate the durations of behaviors, and can be relevant for observing progress or deterioration for health-related issues. 
Soto-Mendoza et al. propose to mine the manually kept logs of geriatric centers for patient monitoring, to derive observations that go beyond the simple content of the logs and to detect anomalies [38]. Some of these annotations can be automatically generated by environmental sensors, or even wearables, but if they are kept by caregivers, they tend to be complex, with temporal gaps, replete with inconsistencies and noise. In their work, about 20.000 activity records were considered for 15 residents of the geriatric center, with 8-9 activities per day per resident. The activities were clustered in an unsupervised fashion, and then each cluster was labeled. A sequential pattern mining approach is used on the labeled events to determine abnormal events.

Sensor activations (or events) collected in an AAL environment are used to detect activities, which require some assumptions to be made [20]. As the semantic level of the description is increased, the labeling and segmentation of the elements of behavior becomes more difficult. For instance, when does idling end, and working begin? This is one difficulty in the assessment of the automatic behavior mining approaches; the labeling inaccuracy reflects both on the quality of the training data, and the specification of the ground truth.

Another major difficulty involves the requirements of testing. Behavior analysis for the elder may require long periods of testing for reliable and robust assessment of performance. For instance in [10], the camera-based classification of sleep patterns shows distinct seasonal trends, as the amount of light in the home environment is different during winter and summer. Shorter amounts of testing, or testing in lab-conditions may produce over-confident estimates of the system's accuracy.

In recent years, the number of mobile applications designed to monitor and maintain elderly has increased sharply. We give a few examples of these in the next section.

\section{Mobile Solutions}

For detecting activities of daily living, phones carry several advantages (and disadvantages) over the more traditional static sensor-based smart environment solutions. Hernández and Favela propose an approach to detect the fatigue of a user with a mobile phone carried in the hip area [15]. The idea is that the accelerometer data can be used for predicting breathing, which is correlated with oxygen consumption and with fatigue. Such an approach removes the need to be in the visual field of a camera for the detection of a certain property or activity. The energy requirement of the phone remains a disadvantage, especially if sensors are supposed to be sampled in a high-frequency, as dictated by the application demands.

Another issue is the cost of installing a dedicated monitoring system in the living environment of the elder, and the maintenance of such a system. Mobile phone based solutions are also sought to minimize the extra hardware cost [15].

In [4], a method is proposed for predicting the next location of an individual based on the observations of his mobility habits. While this problem can be cast into a framework of detecting frequent patterns (see Sect. 3), most learning 
approaches do not quickly adapt to habit changes, for instance due to location changes. Boukhechba et al. propose an incrementally updated tree structure to store the habits of the subject [4]. The association rules derived from the habit tree make prediction possible. Since GPS coordinates are stored continuously on the phone itself, reducing the storage and computation requirements is essential for the usability of such applications.

One should not forget that the evaluation of the interaction itself is not straightforward for elderly. Some difficulties are reported in Hernández and Favela, such as the inconvenience for a subject to attend a lab or similar measurement facility, and the unreliability of self-report measures [15]. Unobtrusive and reliable observation of the subjects is a major issue. Mobile applications can achieve this easily, through the installation or a single application that uses the numerous sensors on the phone. Another benefit is that the application, while monitoring the elder, can take actions and provide notifications, for instance to induce behavior change [32].

In smartphone notifications, different interrupt response patterns are observed depending on the context. In the work of Turner et al., a 6-month in-the-wild case study with over ten thousand to-do list reminders sent to close to a hundred users was performed [40]. The authors propose a four-step model of interrupting the user, where first the device seeks to gain the user's attention by sound, vibration, or visual cues. If the user chooses to react, a summary of the notification is provided to allow the user to either continue the previous activity, or to dedicate some time to the notification. The mobile phone, compared to a smart environment, more readily allows for discrete notifications, and they are handled over a very familiar interface.

\section{Conclusions}

ICT for the elderly has traditionally focused on healthcare aspects, and made extensive use of the rich sensor technology that allows monitoring and engaging subjects in smart environment scenarios [2,26,41]. High-impact applications in these scenarios include fall detection systems [7,25], tools for combatting cognitive decline $[3,17,28]$, and serious games for exercising, rehabilitation and for socializing $[6,11,12,21,30,31,37]$. While we did not tackle them here, robotics research is also active in developing applications usable for the aging population $[5,13,27,34]$.

Recent approaches, as exemplified in this overview paper, focus more on the interaction issues, and take the basic capabilities for granted. Off-the-shelf solutions exist for the building blocks of assistive systems, both in terms of hardware (i.e. inexpensive sensors), middleware, and analysis software [24]. More elaborate scenarios are now being considered, where behavior learning and adaptation is used to solve challenges of noise, idiosyncratic behaviors, and changes over time.

Mobile applications play an increasingly important role in this domain, and offer unobtrusive, interactive and flexible solutions. While issues like power consumption for continuously running applications and compatibility issues remain as open challenges, long-term studies produce promising results, with high acceptability and usability. 
Acknowledgments. This publication was supported by the Dutch national program COMMIT, project VIEWW.

\section{References}

1. Agrawal, R., Srikant, R., et al.: Fast algorithms for mining association rules. In: Proceedings of the 20th International Conference Very Large Data Bases (VLDB), vol. 1215, pp. 487-499 (1994)

2. Alemdar, H., Ersoy, C.: Wireless sensor networks for healthcare: a survey. Comput. Netw. 54(15), 2688-2710 (2010)

3. Bharucha, A.J., Anand, V., Forlizzi, J., Dew, M.A., Reynolds, C.F., Stevens, S., Wactlar, H.: Intelligent assistive technology applications to dementia care: current capabilities, limitations, and future challenges. Am. J. Geriatr. Psychiatry 17(2), 88-104 (2009)

4. Boukhechba, M., Bouzouane, A., Bouchard, B., Gouin-Vallerand, C., Giroux, S.: Online prediction of people's next point-of-interest: concept drift support. In: Salah, A.A., Kröse, B.J., Cook, D.J. (eds.) The Sixth International Workshop on Human Behavior Understanding, pp. 94-115 (2015)

5. Broekens, J., Heerink, M., Rosendal, H.: Assistive social robots in elderly care: a review. Gerontechnology 8(2), 94-103 (2009)

6. Brown, J.A.: Let's play: understanding the role and meaning of digital games in the lives of older adults. In: Proceedings of the International Conference on the Foundations of Digital Games, pp. 273-275. ACM (2012)

7. Chaudhuri, S., Thompson, H., Demiris, G.: Fall detection devices and their use with older adults: a systematic review. J. Geriatr. Phys. Ther. 37(4), 178-196 (2014)

8. Cook, D.J., Das, S.K.: How smart are our environments? an updated look at the state of the art. Pervasive Mobile Comput. 3(2), 53-73 (2007)

9. Derungs, A., Seiter, J., Schuster-Amft, C., Amft, O.: Activity patterns in stroke patients - is there a trend in behaviour during rehabilitation? In: Salah, A.A., Kröse, B.J., Cook, D.J. (eds.) The Sixth International Workshop on Human Behavior Understanding, pp. 143-156 (2015)

10. Eldib, M., Deboeverie, F., Philips, W., Aghajan, H.: Sleep analysis for elderly care using a low-resolution visual sensor network. In: Salah, A.A., Kröse, B.J., Cook, D.J. (eds.) The Sixth International Workshop on Human Behavior Understanding, pp. 25-38 (2015)

11. Gerling, K., Livingston, I., Nacke, L., Mandryk, R.: Full-body motion-based game interaction for older adults. In: Proceedings of the SIGCHI Conference on Human Factors in Computing Systems, pp. 1873-1882. ACM (2012)

12. Gerling, K.M., Schild, J., Masuch, M.: Exergame design for elderly users: the case study of silverbalance. In: Proceedings of the 7th International Conference on Advances in Computer Entertainment Technology, pp. 66-69. ACM (2010)

13. Görer, B., Salah, A.A., Akın, H.L.: A robotic fitness coach for the elderly. In: Augusto, J.C., Wichert, R., Collier, R., Keyson, D., Salah, A.A., Tan, A.-H. (eds.) AmI 2013. LNCS, vol. 8309, pp. 124-139. Springer, Heidelberg (2013)

14. Heinrich, A., Geng, D., Znamenskiy, D., Vink, J., de Haan, G.: Robust and sensitive video motion detection for sleep analysis. IEEE J. Biomed. Health Inform. 18(3), 790-798 (2014) 
15. Hernández, N., Favela, J.: Estimating the perception of physical fatigue among older adults using mobile phones. In: Salah, A.A., Kröse, B.J., Cook, D.J. (eds.) The Sixth International Workshop on Human Behavior Understanding, pp. 81-93 (2015)

16. Jaschinski, C., Ben Allouch, S.: Understanding the user's acceptance of a sensorbased ambient assisted living application. In: Salah, A.A., Kröse, B.J., Cook, D.J. (eds.) The Sixth International Workshop on Human Behavior Understanding, pp. $11-24(2015)$

17. Jorge, J.A.: Adaptive tools for the elderly: new devices to cope with age-induced cognitive disabilities. In: Proceedings of the 2001 EC/NSF Workshop on Universal Accessibility of Ubiquitous Computing: Providing for the Elderly, pp. 66-70. ACM (2001)

18. Kanis, M., Robben, S., Kröse, B.J.: How are you doing? enabling older adults to enrich sensor data with subjective input. In: Salah, A.A., Kröse, B.J., Cook, D.J. (eds.) The Sixth International Workshop on Human Behavior Understanding, pp. 39-51 (2015)

19. Kim, E., Helal, S., Cook, D.: Human activity recognition and pattern discovery. IEEE Pervasive Comput. 9(1), 48-53 (2010)

20. Lago, P., Jiménez-Guarín, C., Roncancio, C.: Contextualized behavior patterns for ambient assisted living. In: Salah, A.A., Kröse, B.J., Cook, D.J. (eds.) The Sixth International Workshop on Human Behavior Understanding, pp. 129-142 (2015)

21. Larsen, L.H., Schou, L., Lund, H.H., Langberg, H.: The physical effect of exergames in healthy elderly - a systematic review. Games Health Res. Dev. Clin. Appl. 2(4), 205-212 (2013)

22. Magnusson, M.S.: Discovering hidden time patterns in behavior: T-patterns and their detection. Behav. Res. Methods Instrum. Comput. 32(1), 93-110 (2000)

23. Mooney, C., Roddick, J.: Sequential pattern mining - approaches and algorithms. ACM Comput. Surv. 45, 19 (2013)

24. Moshnyaga, V., Osamu, T., Ryu, T., Hashimoto, K.: Identification of basic behavioral activities by heterogeneous sensors of in-home monitoring system. In: Salah, A.A., Kröse, B.J., Cook, D.J. (eds.) The Sixth International Workshop on Human Behavior Understanding, pp. 157-170 (2015)

25. Mubashir, M., Shao, L., Seed, L.: A survey on fall detection: principles and approaches. Neurocomputing 100, 144-152 (2013)

26. Osmani, V., Balasubramaniam, S., Botvich, D.: Human activity recognition in pervasive health-care: supporting efficient remote collaboration. J. Netw. Comput. Appl. 31(4), 628-655 (2008). http://www.sciencedirect.com/science/article/pii/ S1084804507000719

27. Pineau, J., Montemerlo, M., Pollack, M., Roy, N., Thrun, S.: Towards robotic assistants in nursing homes: challenges and results. Robot. Auton. Sys. 42(3), 271-281 (2003)

28. Pollack, M.E.: Intelligent technology for an aging population: the use of ai to assist elders with cognitive impairment. AI Mag. 26(2), 9 (2005)

29. Ranjan, J., Whitehouse, K.: Rethinking the fusion of technology and clinical practices in functional behavior analysis for the elderly. In: Salah, A.A., Kröse, B.J., Cook, D.J. (eds.) The Sixth International Workshop on Human Behavior Understanding, pp. 52-65 (2015)

30. Rego, P., Moreira, P.M., Reis, L.P.: Serious games for rehabilitation: a survey and a classification towards a taxonomy. In: 2010 5th Iberian Conference on Information Systems and Technologies (CISTI), pp. 1-6. IEEE (2010) 
31. Rice, M., Wan, M., Foo, M.H., Ng, J., Wai, Z., Kwok, J., Lee, S., Teo, L.: Evaluating gesture-based games with older adults on a large screen display. In: Proceedings of the 2011 ACM SIGGRAPH Symposium on Video Games, pp. 17-24. ACM (2011)

32. Salah, A.A., Lepri, B., Pianesi, F., Pentland, A.S.: Human behavior understanding for inducing behavioral change: application perspectives. In: Salah, A.A., Lepri, B. (eds.) HBU 2011. LNCS, vol. 7065, pp. 1-15. Springer, Heidelberg (2011)

33. Salah, A.A., Pauwels, E., Tavenard, R., Gevers, T.: T-patterns revisited: mining for temporal patterns in sensor data. Sensors 10(8), 7496-7513 (2010)

34. Schulz, R., Wahl, H.W., Matthews, J.T., Dabbs, A.D.V., Beach, S.R., Czaja, S.J.: Advancing the aging and technology agenda in gerontology. The Gerontologist p. gnu071 (2014)

35. Selwyn, N.: The information aged: a qualitative study of older adults' use of information and communications technology. J. Aging Stud. 18(4), 369-384 (2004)

36. Selwyn, N., Gorard, S., Furlong, J., Madden, L.: Older adults' use of information and communications technology in everyday life. Ageing Soc. 23(05), 561-582 (2003)

37. Shim, N., Baecker, R., Birnholtz, J., Moffatt, K.: Tabletalk poker: an online social gaming environment for seniors. In: Proceedings of the International Academic Conference on the Future of Game Design and Technology, pp. 98-104. ACM (2010)

38. Soto-Mendoza, V., Beltrán, J., Chávez, E., Hernández, J., García-Macías, J.A.: Abnormal behavioral patterns detection from activity records of institutionalized older adults. In: Salah, A.A., Kröse, B.J., Cook, D.J. (eds.) The Sixth International Workshop on Human Behavior Understanding, pp. 116-128 (2015)

39. Sutton, C., McCallum, A.: An introduction to conditional random fields for relational learning. In: Introduction to Statistical Relational Learning, pp. 93-128 (2006)

40. Turner, L.D., Allen, S.M., Whitaker, R.M.: Push or delay? decomposing smartphone notification response behaviour. In: Salah, A.A., Kröse, B.J., Cook, D.J. (eds.) The Sixth International Workshop on Human Behavior Understanding, pp. 66-80 (2015)

41. Varshney, U.: Pervasive healthcare and wireless health monitoring. Mobile Netw. Appl. 12(2-3), 113-127 (2007)

42. Visser, T., Vastenburg, M.H., Keyson, D.V.: Designing to support social connectedness: the case of snowglobe. Int. J. Des. 5(3), 129-142 (2011)

43. White, H., McConnell, E., Clipp, E., Bynum, L., Teague, C., Navas, L., Craven, S., Halbrecht, H.: Surfing the net in later life: a review of the literature and pilot study of computer use and quality of life. J. Appl. Gerontol. 18(3), 358-378 (1999) 